\title{
Byz-GentleRain: An Efficient Byzantine-tolerant Causal Consistency Protocol
}

\author{
Kaile Huang ${ }^{1}$, Hengfeng $\mathrm{Wei}^{2,3 \star}$, Yu Huang ${ }^{2}$, Haixiang $\mathrm{Li}^{4}$, and Anqun $\mathrm{Pan}^{4}$ \\ 1 Computer Science and Technology, Nanjing University, China \\ MG1933024@smail.nju.edu.cn \\ 2 State Key Laboratory for Novel Software Technology, Nanjing University, China \\ $\{$ hfwei, yuhuang $\} @$ ju.edu.cn \\ 3 Software Institute, Nanjing University, China \\ 4 Tencent Inc., China \\ \{blueseali, aaronpan\}@tencent.com
}

\begin{abstract}
Causal consistency is a widely used weak consistency model that allows high availability despite network partitions. There are plenty of research prototypes and industrial deployments of causally consistent distributed systems. However, as far as we know, none of them consider Byzantine faults, except Byz-RCM proposed by Tseng et al. Byz-RCM achieves causal consistency in the client-server model with $3 f+1$ servers where up to $f$ servers may suffer Byzantine faults, but assumes that clients are non-Byzantine. In this work, we present Byz-Gentlerain, the first causal consistency protocol which tolerates up to $f$ Byzantine servers among $3 f+1$ servers in each partition and any number of Byzantine clients. Byz-GentleRain is inspired by the stabilization mechanism of GentleRain for causal consistency. To prevent causal violations due to Byzantine faults, Byz-GentleRain relies on PBFT to reach agreement on a sequence of global stable times and updates among servers, and only updates with timestamps less than or equal to such common global stable times are visible to clients. We prove that Byz-GentleRain achieves Byz$\mathrm{CC}$, the causal consistency variant in the presence of Byzantine faults. We evaluate Byz-GentleRain on Aliyun. The preliminary results show that Byz-GentleRain is efficient on typical workloads.
\end{abstract}

Keywords: Causal consistency · Byzantine faults · PBFT · GentleRain - Byz-GentleRain.

\section{Introduction}

For high availability and low latency even under network partitions, distributed systems often partition and replicate data among multiple nodes 7]. Due to the CAP theorem [6] many distributed systems choose to sacrifice strong consistency and to implement weak ones.

Causal consistency 1 is one of the most widely used consistency model in distributed systems. There are several variants of causal consistency in the

\footnotetext{
* Corresponding Author
} 
literature 1, 1, 11, 18. They all guarantee that an update does not become visible until all its causality are visible. We informally explain it in the "Lost-Ring" example [13. Alice first posts that she has lost her ring. After a while, she posts that she has found it. Bob sees Alice's two posts, and comments that "Glad to hear it". We say that there is a read-from dependency from Alice's second post to Bob's get operation, and a session dependency from Bob's get operation to his own comment. By transitivity, Bob's comment causally depend on Alice's second post. Thus, when Carol, a friend of Alice and Bob, sees Bob's comment, she should also see Alice's second post. If she saw only Alice's first post, she would mistakenly think that Bob is glad to hear that Alice has lost her ring.

There are plenty of research prototypes and industrial deployments of causally consistent distributed systems (e.g., COPS [16], Eiger 17], GentleRain 99, Cure 2], MongoDB [20], and Byz-RCM [19]). GentleRain uses a stabilization mechanism to make updates visible while respecting causal consistency. It timestamps all updates with the physical clock value of the server where they originate. Each server $s$ periodically computes a global stable time gst, which is a lower bound on the physical clocks of all servers. This ensures that no updates with timestamps $\leq$ gst will be generated. Thus, it is safe to make the updates with timestamps $\leq$ gst at $s$ visible to clients. A get operation with dependency time $d t$ issued to $s$ will wait until gst $\geq d t$ and then obtain the latest version before gst.

However, none of these causal consistency protocols/systems consider Byzantine faults, except Byz-RCM (Byzantine Resilient Causal Memory) in [19]. ByzRCM achieves causal consistency in the client-server model with $3 f+1$ servers where up to $f$ servers may suffer Byzantine faults, and any number of clients may crash. Byz-RCM has also been shown optimal in terms of failure resilience. However, Byz-RCM did not tolerate Byzantine clients, and thus it could rely on clients' requests to identify bogus requests from Byzantine servers 19 .

In this work, we present Byz-GentleRain, the first Byzantine-tolerant causal consistency protocol which tolerates up to $f$ Byzantine servers among $3 f+1$ servers in each partition and any number of Byzantine clients. It uses PBFT [8] to reach agreement among servers on a total order of client requests. The major challenge Byz-GentleRain faces is to ensure that the agreement is consistent with the causal order. To this end, Byz-GentleRain should prevent causality violations caused by Byzantine clients or servers: Byzantine clients may violate the session order by fooling some servers that a request happened before another that was issued earlier. Byzantine servers may forge causal dependencies by attaching arbitrary metadata for causality tracking to the forward messages. To migrate the potential damages of Byzantine servers, we let clients assign totally ordered timestamps to updates in Byz-GentleRain. Utilizing the digital signatures mechanism, Byzantine servers cannot forge causal dependencies.

To preserve causality, Byz-GentleRain uses the stabilization mechanism of GentleRain. As explained above, the timestamps in Byz-GentleRain are generated by clients. However, it is unrealistic to compute a lower bound on physical clock values of an arbitrary number of clients. Therefore, each server $s$ in ByzGentleRain maintains and periodically computes a global stable time gst which 


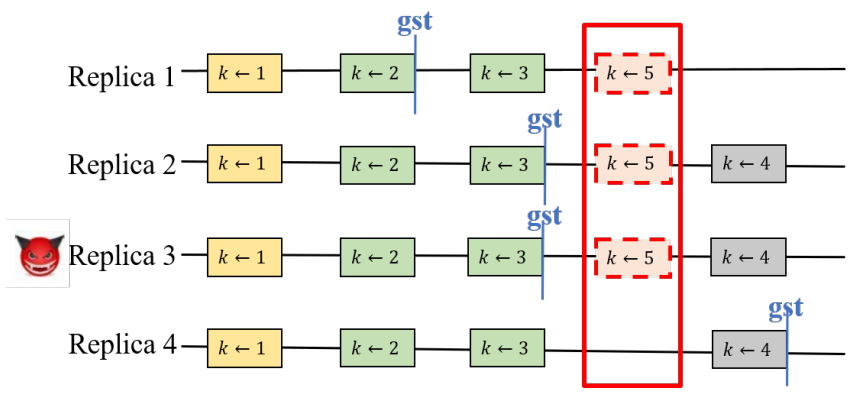

Fig. 1: Why the servers in Byz-GentleRain need to synchronize global stable times.

is a lower bound on physical clock values of the clients it is aware of. In the following, we argue that simply refusing any updates with timestamps $\leq$ gst on each server as GentleRain does may lead to causality violations. Consider a system of four servers which are replicas all maintaining a single key $k$, as shown in Figure 1. Due to asynchrony, these four servers may have different values of gst. Without loss of generality, we assume that gst $1<$ gst $_{2}=$ gst $_{3}<$ gst $_{4}$, as indicated by vertical lines. Now suppose that a new update $u: k \leftarrow 5$ with timestamp between gst ${ }_{3}$ and gst $_{4}$ arrives, and we want to install it on $\geq 3$ servers, using quorum mechanism. In this scenario, if each server refuses any updates with timestamps smaller than or equal to its gst, the update $u$ can only be accepted by the first 3 servers, indicated by dashed boxes. Suppose that server 3 is a Byzantine server, which may expose or hide the update $u$ as it will. Consequently, later read operations which read from $\geq 3$ servers may or may not see this update $u$. That is, the Byzantine server 3 may cause causality violations.

To cope with this problem, we need to synchronize the global stable times of servers. When a server periodically computes its gst, it checks whether no larger global stable time has been or is being synchronized. If so, the server will try to synchronize its gst among all servers, by running PBFT independently in each partition. For each partition, the PBFT leader is also responsible for collecting updates with timestamps $\leq$ gst from $2 f+1$ servers, and synchronizing them on all servers. Once successfully synchronized, a global stable time becomes a common global stable time, denoted cgst, and in each partition the updates with timestamps $\leq$ cgst on all correct servers are the same. Therefore, each server can safely refuse any updates with timestamps smaller than or equal to its cgst.

Still, the classic PBFT is insufficient to guarantee causality, since a Byzantine leader of each partition may propose an arbitrary set of updates. To avoid this, in Byz-GentleRain the PBFT leader will also include the sets of updates it collects from $2 f+1$ servers in its PROPOSE message. A server will reject the PROPOSE message if it finds the contents of this message have been manipulated by checking hash and signatures.

Thus, we make the following contributions: 
Table 1: Notations.

\begin{tabular}{|c|c|}
\hline Notations & Meaning \\
\hline $\bar{D}$ & number of data centers \\
\hline$P$ & number of partitions \\
\hline$r_{d}^{p}$ & the replica of partition $p$ in data center $d$ \\
\hline$\overline{\operatorname{PARTITION}(k)}$ & the partition that holds key $k$ \\
\hline $\operatorname{REPLICAS}(p)$ & the set of replicas of partition $p$ \\
\hline DATACENTER $(d)$ & the set of servers in data center $d$ \\
\hline $\mathcal{S}$ & the set of all $D \times P$ servers in the key-value store \\
\hline clock $_{c}$ & clock at client $c$ \\
\hline $\mathrm{dt}_{c}$ & dependency time at client $c$ \\
\hline cgst $_{c}$ & the maximum common global stable time known by client $c$ \\
\hline clock $_{d}^{p}$ & clock at replica $r_{d}^{p}$ \\
\hline $\mathrm{Ist}_{d}^{p}$ & local stable time at replica $r_{d}^{p}$ \\
\hline $\mathrm{gst}_{d}^{p}$ & global stable time at replica $r_{d}^{p}$ \\
\hline $\operatorname{cgst}_{d}^{p}$ & common global stable time at replica $r_{d}^{p}$ \\
\hline curr_cgst $_{d}^{p}$ & temporary common global stable time at replica $r_{d}^{p}$ during PBFT \\
\hline $\begin{array}{l}\mathrm{VV}_{d}^{p} \\
\mathrm{LV}_{d}^{p}\end{array}$ & $\begin{array}{l}\text { version vector at replica } r_{d}^{p} \\
\text { local stable time vector at replica } r_{d}^{p}\end{array}$ \\
\hline Key & the set of keys, ranged over by $k$ \\
\hline Val & the set of values, ranged over by $v$ \\
\hline V Val & the set of versioned values, ranged over by $v v$ \\
\hline store $_{d}^{p} \subseteq \mathrm{VVal}$ & store maintained at replica $r_{d}^{p}$ \\
\hline STORE & the union of stores at all replicas, that is, STORE $\triangleq \bigcup_{1<i<D, 1<j<P}$ store $_{i}^{j}$ \\
\hline
\end{tabular}

- We define Byzantine Causal Consistency (Byz-CC), which is a causal consistency variant in the presence of Byzantine faults (Section 3).

- We present Byz-GentleRain, the first Byzantine-tolerant causal consistency protocol. It tolerates up to $f$ Byzantine servers among $3 f+1$ ones and any number of Byzantine clients (Section 4). All reads and updates complete in one round-trip.

- We evaluate Byz-GentleRain on Aliyun. The preliminary results show that Byz-GentleRain is efficient on typical workloads (Section 5 ).

Section 2 describes the system model and failure model. Section 6 discusses related work. Section 7 concludes the paper. The proofs can be found in Appendix $\mathrm{A}$.

\section{Model}

We adopt the client/server architecture 15,19], in which each client or server has its unique id. Table 1 summarizes the notations used in this paper.

\subsection{System Model}

We consider a distributed multi-version key-value store, which maintains keys in the set Key (ranged over by $k$ ) with values in the set Val (ranged over by $v$ ). 
Each value is associated with a unique version, consisting of the timestamp of the update which creates this version and the id of the client which issues this update. We denote by $\mathrm{VVal}$ (ranged over by $v v$ ) the set of versioned values.

The distributed key-value store runs at $D$ data centers, each of which has a full copy of data. In each data center, the full data is sharded into $P$ partitions. For a key $k \in$ Key, we use PARTITION $(k)$ to denote the partition that holds $k$. Each partition is replicated across $D$ data centers. For a partition $p$, we use $\operatorname{REplicas}(p)$ to denote the set of replicas of $p$. For a data center $d$, we use DAtaCEnTER $(d)$ to denote the set of servers in $d$. We denote by $r_{d}^{p}$ the replica of partition $p$ at data center $d$. We denote by $\mathcal{S}$ the set of all $D \times P$ servers in the key-value store. For each individual partition $p$, we call a set $Q$ of $2 f+1$ replicas in $\operatorname{REPLICAS}(p)$ a quorum and denote it by quorum $(Q)$.

For convenience, we model the key-value store at replica $r_{d}^{p}$, denoted store ${ }_{d}^{p}$, by a set of (unique) versioned values. That is, store ${ }_{d}^{p} \subseteq$ VVal. We denote by STORE the union of stores at all replicas. That is, $\mathrm{STORE} \triangleq \bigcup_{1 \leq i \leq D, 1 \leq j \leq P}$ store $_{i}^{j}$. The distributed key-value store offers two operations to clients:

- GET $(k)$. A get operation which returns the value of some version of key $k$.

- $\operatorname{PUT}(k, v)$. A put operation which updates key $k$ with value $v$. This creates a new version of $k$.

We assume that each client or server is equipped with a physical clock, which is monotonically increasing. Clocks at different clients are loosely synchronized by a protocol such as NTP ${ }^{5}$ The correctness of Byz-GentleRain does not depend on the precision of clock synchronization, but large clock drifts may negatively impact its performance.

\section{$2.2 \quad$ Failure Model}

Clients and servers are either correct or faulty. Correct clients and servers obey their protocols, while faulty ones may exhibit Byzantine behaviors [8], by deviating arbitrarily from their protocols.

We assume asynchronous point-to-point communication channels among clients and servers. Messages may be delayed, duplicated, corrupted, or delivered out of order. We do not assume known bounds on message delays. The communication network is fully connected. We require that if the two ends of a channel are both correct and the sender keeps retransmitting a message, then the message can eventually be delivered.

We also assume the channels are authenticated. Clients and servers can sign messages using digital signatures when needed. A message $m$ signed by a client $c$ or a replica $r_{d}^{p}$ is denoted by $\langle m\rangle_{c}$ or $\langle m\rangle_{d}^{p}$, respectively. We denote by $\operatorname{valid}(m)$ that $m$ is valid in signatures. We also use a cryptographic hash function hash(), which is assumed to be collision-resistant: the probability of an adversary producing inputs $m$ and $m^{\prime}$ such that hash $(m)=$ hash $\left(m^{\prime}\right)$ is negligible [5,8].

\footnotetext{
${ }^{5}$ NTP: The Network Time Protocol. http://www.ntp.org/
} 


\section{Byzantine Causal Consistency}

Causal consistency variants in the literature 1, 1, 11, 18 are defined based on the happens-before relation among events [12. However, they are not applicable to systems that allows Byzantine nodes, particularly Byzantine clients. We now adapt the happens-before relation in Byzantine-tolerant systems, and define Byzantine Causal Consistency (Byz-CC) as follows. For two events $e$ and $f$, we say that $e$ happens before $f$, denoted $e \rightsquigarrow f$, if and only if one of the following three rules holds:

- Session-order. Events $e$ and $f$ are two operation requests issued by the same correct client, and $e$ is issued before $f$. We denote it by $e \stackrel{\text { so }}{\rightarrow} f$. We do not require session order among operations issued by Byzantine clients.

- Read-from relation. Event $e$ is a PUT request issued by some client and $f$ is a GET request issued by a correct client, and $f$ reads the value updated by $e$. We denote it by $e \stackrel{\text { rf }}{\rightarrow} f$. Since a GET of Byzantine clients may return an arbitrary value, we do not require read-from relation induced by it.

- Transitivity. There is another operation request $g$ such that $e \rightsquigarrow g$ and $g \rightsquigarrow f$.

If $e \rightsquigarrow f$, we also say that $f$ causally depends on $e$ and $e$ is a causal dependency of $f$. A version $v v$ of a key $k$ causally depends on version $v v^{\prime}$ of key $k^{\prime}$, if the update of $v v$ causally depends on that of $v v^{\prime}$. A key-value store satisfies Byz-CC if, when a certain version of a key is visible to a client, then so are all of its causal dependencies.

\section{The Byz-GentleRain Protocol}

As discussed in Section 1 it is the clients in Byz-GentleRain that are responsible for generating totally ordered timestamps for updates. Specifically, when a client issues an update, it assigns to the update a timestamp consisting of its current clock and identifier.

As in GentleRain, we also distinguish between the updates that have been received by a server and those that have been made visible to clients. ByzGentleRain guarantees that an update can be made visible to clients only if so are all its causal dependencies. The pseudocode in Algorithms 13 dealing with Byzantine faults is underlined.

\subsection{Key Designs}

In Byz-GentleRain, both clients and servers maintain a common global stable time cgst. We denote the cgst at client $c$ by $\operatorname{cgst}_{c}$ and that at replica $r_{d}^{p}$ by $\operatorname{cgst}_{d}^{p}$. We maintain the following invariants that are key to the correctness of Byz-GentleRain:

INV (I): Consider $\operatorname{cgst}_{c}$ at any time $\sigma$. All updates issued by correct client $c$ after time $\sigma$ have a timestamp $>$ cgst $_{c}$. 


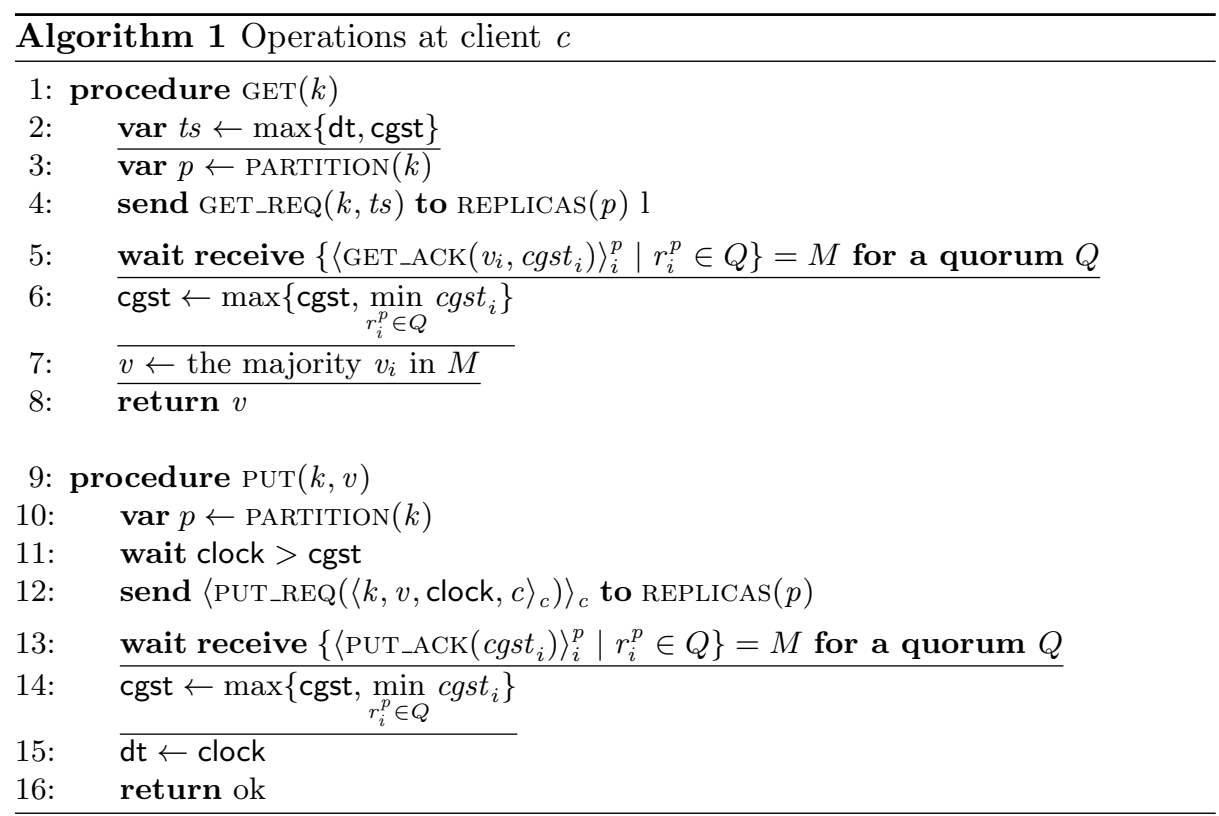

INV (II): Consider $\operatorname{cgst}_{d}^{p}$ at any time $\sigma$. No updates with timestamps $\leq \operatorname{cgst}_{d}^{p}$ will be successfully executed at $>f$ correct replicas in $\operatorname{REPLiCAS}(p)$ after time $\sigma$.

INV (III): Consider a cgst value. For any two correct replicas $r_{d}^{p}$ and $r_{i}^{p}$ (where $i \neq d$ ) of partition $p$, if $\operatorname{cgst}_{d}^{p} \geq$ cgst and $\operatorname{cgst}_{i}^{p} \geq$ cgst, then the updates with timestamps $\leq$ cgst in store ${ }_{d}^{p}$ and store ${ }_{i}^{p}$ are the same.

Byz-GentleRain further enforces the following rules for reads and updates:

RULE (I): For a correct replica $r_{d}^{p}$, any updates with timestamps $>\operatorname{cgst}_{d}^{p}$ in store $_{d}^{p}$ are invisible to any clients.

RULE (II): Any correct replica $r_{d}^{p}$ will reject any updates with timestamps $\leq \operatorname{cgst}_{d}^{p}$.

RULE (III): For a read operation with timestamp $t s$ issued by client $c$, any correct replica $r_{d}^{p}$ that receives this operation must wait until $\operatorname{cgst}_{d}^{p} \geq$ $t s$ before it returns a value to client $c$.

In the following sections, we explain how these invariants and rules are implemented and why they are important to the correctness.

\subsection{Client Operations}

To capture the session order, each client maintains a dependency time $\mathrm{dt}$, which is the clock value of its last put operation. When a client $c$ issues a GET operation on key $k$, it first takes as ts the minimum of its dependency time $\mathrm{dt}_{c}$ and common global stable time cgst $_{c}$ (line 1 12 . Then it sends a GET_REQ request with ts 


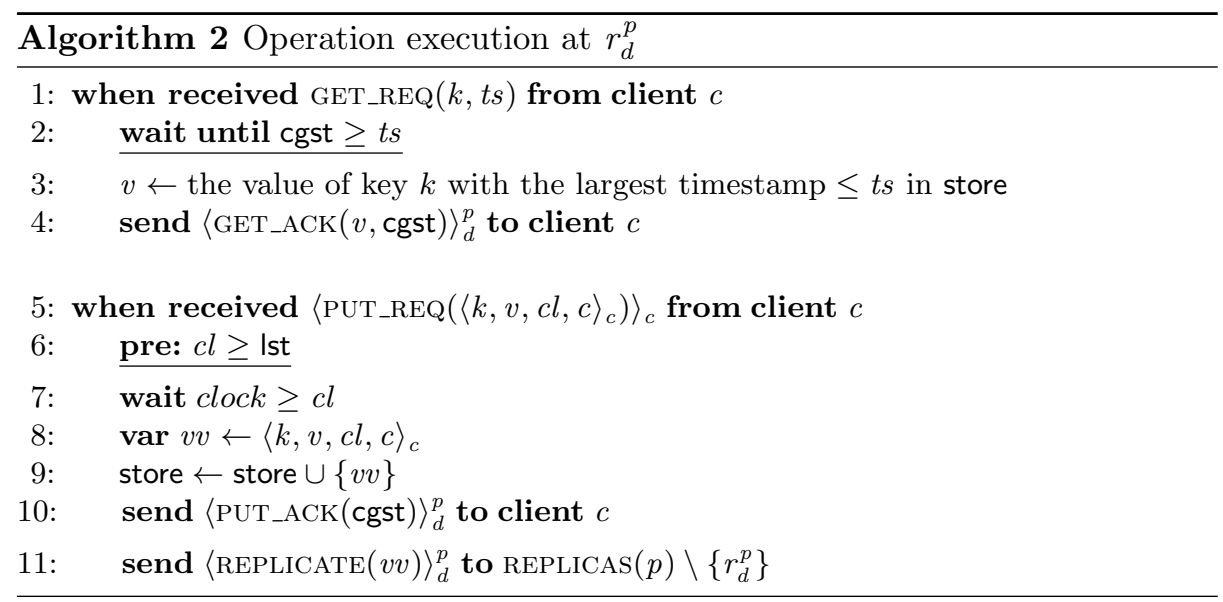

to $\operatorname{REPlicas}(p)$ (line 14), where $p$ is the partition holding $k$ (line 13 ). Next, the client waits to receive a set $M$ of GET_ACK responses from a quorum $Q$ of $\operatorname{REPLICAS}(p)$ (line 15 . For each replica $r_{i}^{p} \in Q$, the GET_ACK response from $r_{i}^{p}$ carries a value $v_{i}$ of key $k$ and its common global stable time cgst $_{i}$ when $r_{i}^{p}$ computes the value $v_{i}$ to return (line 23, discussed in Section 4.3). The client takes the minimum cgst $_{i}$ for $r_{i}^{p} \in Q$, and uses it to update cgst $_{c}$ if the latter is smaller (line 16).

Since there are at most $f$ Byzantine replicas in a partition, at least $f+1$ GET_ACK responses are from correct replicas. By RuLE (III) and INV (III), these responses from correct replicas contain the same value, denoted $v$. Hence, $v$ is the majority $v_{i}$ in $M$ (line 17). Finally, the client returns $v$ (line 18 ).

When a client $c$ issues a PUT operation on key $k$ with value $v$, it sends a PUT_REQ request carrying its clock $c$ and id $c$ to $\operatorname{REPLICAS}(p)$ (line 112, where $p$ is the partition holding $k$ (line 110 . Next the client waits to receive a set $M$ of PUT_ACK responses from a quorum $Q$ of $\operatorname{REPLICAS}(p)$ (line 113). For each replica $r_{i}^{p} \in Q$, the PUT_ACK response from $r_{i}^{p}$ carries its common global stable time cgst $_{i}$. Then, the client takes the minimum cgst $_{i}$ for $r_{i}^{p} \in Q$, and uses it to update cgst if the latter is smaller (line 114). Finally, $\mathrm{dt}_{c}$ is set to the current clock $_{c}$ (line 115).

\subsection{Operation Executions at Replicas}

When a replica $r_{d}^{p}$ receives a $\operatorname{GET} \_\operatorname{REQ}(k, t s)$ request from some client $c$, it first waits until cgst $\geq t$ s (line 22 where $t s \triangleq \max \left\{\mathrm{dt}_{c}, \mathrm{cgst}_{c}\right\}$ (line 12 ). This implements RULE III] and is used to ensure the session guarantee on client $c$ and eventual visibility of updates to $c$. Then the replica obtains the value $v$ of key $k$ in store which has the largest timestamp before $t s$, breaking ties with client ids (line 2 3). Finally, it sends a signed GET_ACK response, along with the value $v$ and its current cgst, to client $c$ (line 24 . 
When a replica $r_{d}^{p}$ receives a $\operatorname{PUT}$ _REQ $(k, v, c l, c)$ request from client $c$, it first checks the precondition $c l \geq$ curr_cgst $_{d}^{p}$ (line 26). This enforces RuLE II. and prevents fabricated updates with timestamps $\leq$ cgst at store ${ }_{d}^{p}$ from now on. If the precondition holds, the replica adds the new versioned version $v v \triangleq$ $\langle k, v, c l, c\rangle_{c}$ signed by $c$ (line 28) to store ${ }_{d}^{p}$ (line 29). Then, the replica sends a signed PUT_ACK response, with its $\operatorname{cgst}_{d}^{p}$, to client $c$ (line 2 10. Finally, it broadcasts a signed REPLICATE message with $v v$ to other replicas in partition $p$ (line 211).

\subsection{Metadata}

Replica States Each replica $r_{d}^{p}$ maintains a version vector $\mathbf{V}_{d}^{p}$ of size $D$, with each entry per data center. For data center $d, \mathbf{V}_{d}^{p}[d]$ is the timestamp of the last update that happens at $r_{d}^{p}$. For data center $i \neq d, \mathrm{VV}_{d}^{p}[i]$ is the largest timestamp of the updates that happened at replica $r_{i}^{p}$ and have been propagated to $r_{d}^{p}$. For fault-tolerance, we compute the local stable time $\mathrm{Ist}_{d}^{p}$ at replica $r_{d}^{p}$ as the $(f+1)$-st minimum element of its $\mathrm{VV}_{d}^{p}$.

Each replica $r_{d}^{p}$ also maintains a lst vector $\mathrm{LV}_{d}^{p}$ of size $P$, with each entry per partition. For partition $1 \leq j \leq P, \operatorname{LV}_{d}^{p}[j]$ is the largest lst of replica $r_{d}^{j}$ of which $r_{d}^{p}$ is aware. We compute the global stable time gst $_{d}^{p}$ at replica $r_{d}^{p}$ as the minimum element of its $\operatorname{LV}_{d}^{p}$. That is, $\operatorname{gst}_{d}^{p} \triangleq \min _{1 \leq j \leq P} \operatorname{LV}_{d}^{p}[j]$.

Each replica $r_{d}^{p}$ periodically synchronizes their $g_{s} t_{d}^{p}$ with others via PBFT, and maintains a common global stable time $\operatorname{cgst}_{d}^{p}$. We discuss it in Section 4.5.

Propagation As in GentleRain, Byz-GentleRain propagates and updates metadata in the background. Once a new version $v v$ is created at replica $r_{d}^{p}$, the replica sends a signed REPLICATE $(v v)$ message to other replicas of partition $p$ (line 211 .

When replica $r_{d}^{p}$ receives a $\operatorname{REPLICATE}(v v)$ message from another replica $r_{i}^{p}$ in data center $i \neq d$, it stores $v v$ in its store ${ }_{d}^{p}$ (line 32 , and updates $\mathrm{VV}_{d}^{p}[i]$ to vv.cl if the latter is larger (line 33 .

Each replica $r_{d}^{p}$ periodically computes its $\mathrm{Ist}_{d}^{p}$ (line 35 and sends a signed $\operatorname{BROADCAST}\left(\operatorname{Ist}_{d}^{p}\right)$ message to DATACENTER $(d)$, all the servers in data center $d$ (line 36 ).

When replica $r_{d}^{p}$ receives a $\operatorname{BROADCAST}(l s t)$ message from another replica $r_{d}^{j}$ in data center $d$, it updates $\mathrm{LV}_{d}^{p}$ and gst $_{d}^{p}$ accordingly (lines 38 and 39. . If the new gst $_{d}^{p}$ is larger than $\operatorname{cgst}_{d}^{p}$ (line 310 , the replica $r_{d}^{p}$ sends a signed NEW_CGST $\left(\right.$ gst $\left._{d}^{p}\right)$ message to all servers $\mathcal{S}$ of the key-value store (line 311 .

To ensure liveness, a replica $r_{d}^{p}$ periodically (e.g., at time interval $\Delta ;$ line 313 ) sends a signed $\operatorname{HB}\left(\operatorname{clock}_{d}^{p}\right)$ heartbeat to $\operatorname{REPLICAS}(p)$ (line 314 . When replica $r_{d}^{p}$ receives a heartbeat $\mathrm{HB}($ clock $)$ message from replica $r_{i}^{p}$, it updates its $\mathrm{VV}_{d}^{p}[i]$ to clock if the latter is larger (line 316 ).

\subsection{Synchronization of Global Stable Time}

Each individual partition $p$ independently runs PBFT 8 to reach agreement on a common global stable time cgst and the same set of updates before cgst across 


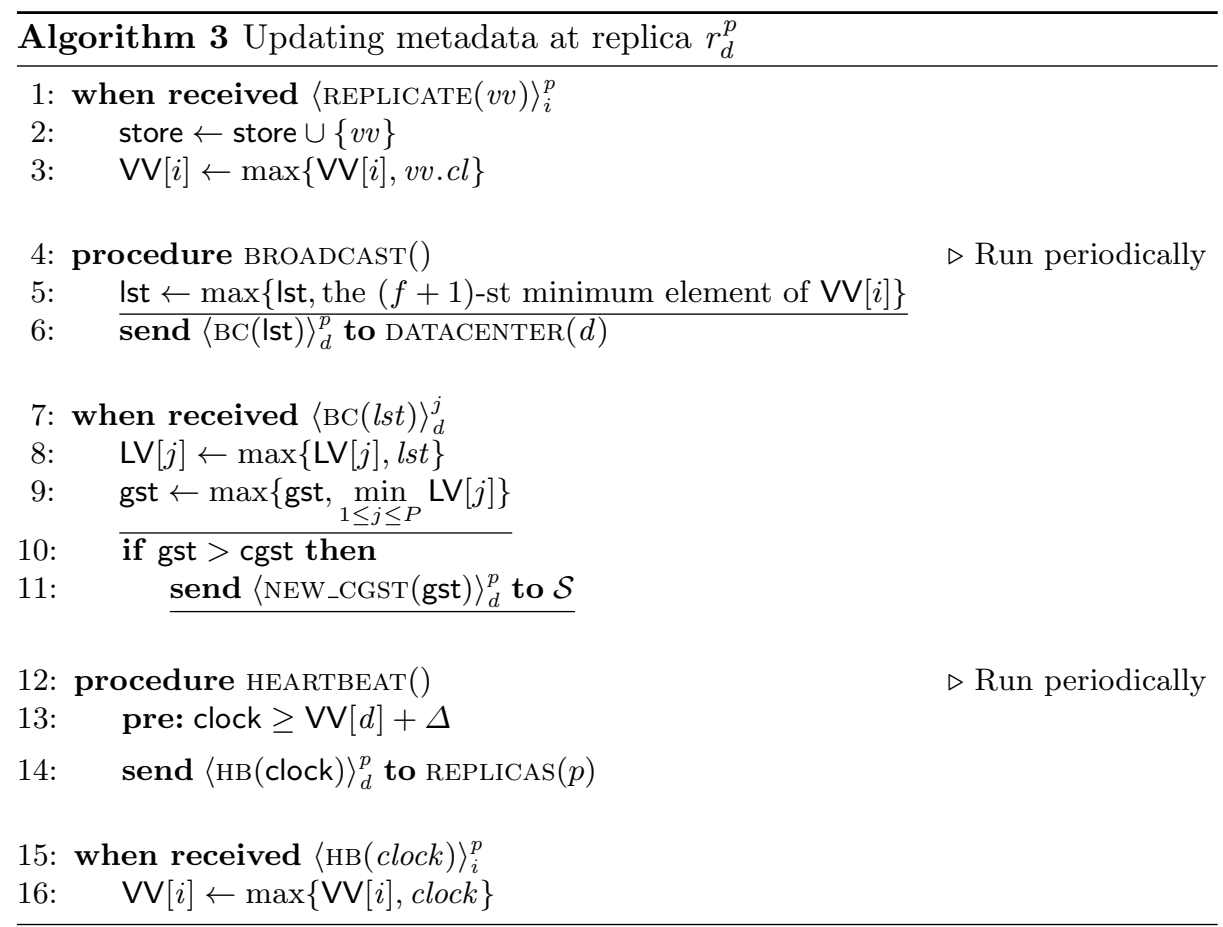

$\operatorname{REPLICAS}(p)$ (Algorithm 4). We follow the pseudocode of single-shot PBFT described in [5], and refer its detailed description and correctness proof to [5]. In the following, we elaborate the parts specific to synchronization of global stable time; see the pseudocode underlined in Algorithm 4.

When replica $r_{d}^{p}$ receives a NEW_CGST $(g s t)$ message, it first checks whether $g s t \leq \operatorname{lst}_{d}^{p}$ as expected and gst $>$ curr_cgst $_{d}^{p}$ which means that no smaller global stable time has been or is being synchronized (line 4/2). If so, it sets curr_cgst to gst (line 43). Now the replica stops accepting updates with timestamps < curr_cgst $_{d}^{p}$ (line 2 6). Then it triggers a NEWviEW action with a view larger than curr_view $_{d}^{p}$ (line 44 ).

As in classic PBFT 5, 8, the NEWVIEW(view) action can also be triggered spontaneously, due to timeout, or by failure detectors. When it is triggered at a replica $r_{d}^{p}$, the replica will send a signed NEWLEADER message to the leader leader (view) of view in $\operatorname{REPLICAS}(p)$ (line 410). The NEWLEADER message carries both curr_cgst ${ }_{d}^{p}$ and prepared_store ${ }_{d}^{p}$ which is the set of updates collected in prepared_view ${ }_{d}^{p}$.

When replica $r_{d}^{p}$ receives a set $M$ of NEWLEADER messages from a quorum $Q$ of $\operatorname{Replicas}(p)$, it selects as its proposal from $M$ the set store $_{j}$ of collected updates that is prepared in the highest view, say view ${ }_{i}$ (line 415), or, if there are no such $s t_{0}{ }_{j}$, its own proposal. In the latter case, the replica sets its curr_cgst ${ }_{d}^{p}$ to the maximum of $c g s t_{i}$ in $Q$ that are $\leq \operatorname{lst}_{d}^{p}$ (line 417 ). Then, it sends a signed 


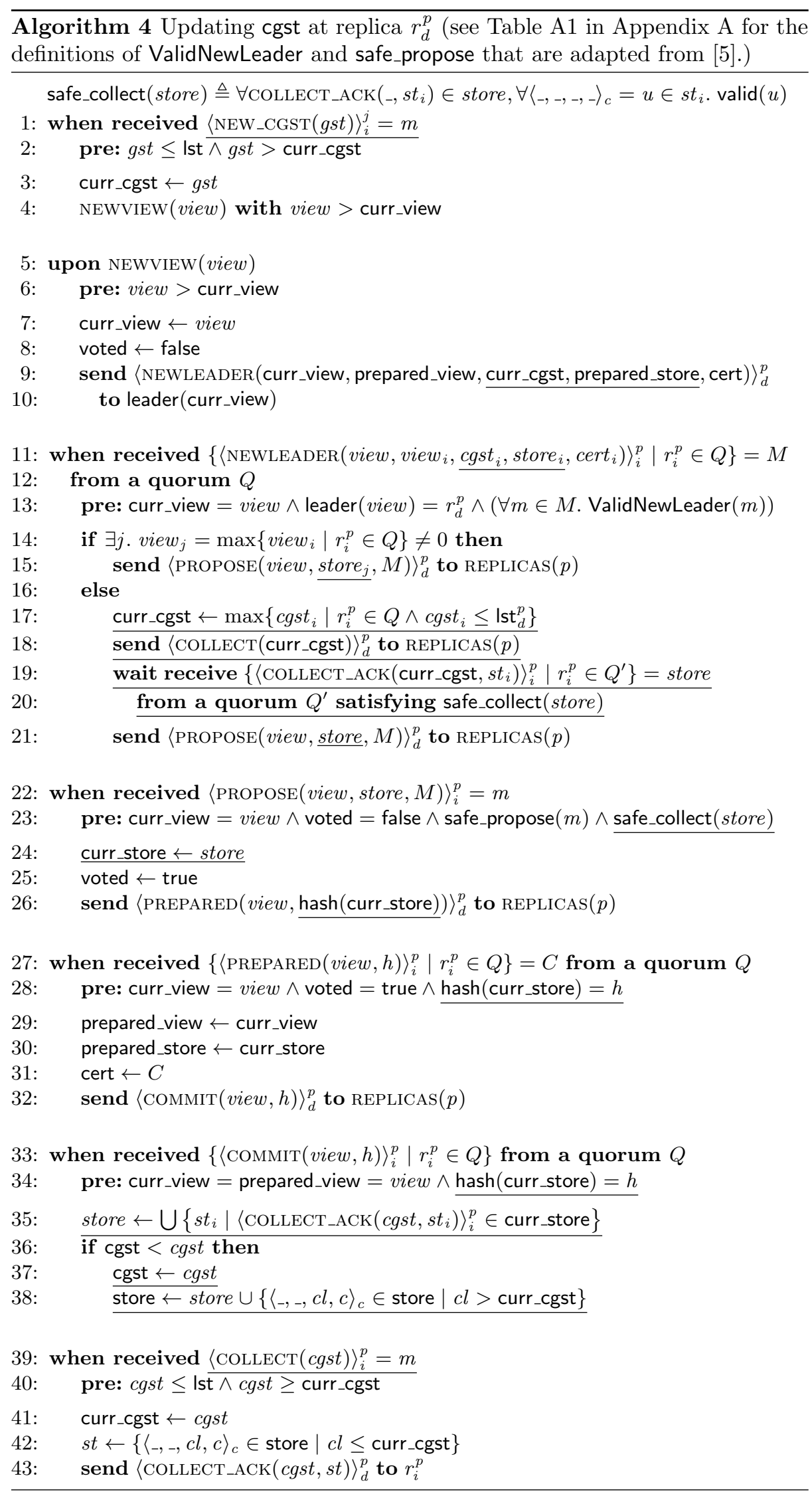


$\operatorname{COLLECT}\left(\right.$ curr_cgst $\left._{d}^{p}\right)$ message to $\operatorname{REPLICAS}(p)$ (line 318), and waits to receive enough COLLECT_ACK messages.

When replica $r_{d}^{p}$ receives a COLLECT $(c g s t)$ message from replica $r_{i}^{p}$ and $c g s t$ passes the precondition (line 440), it first sets its curr_cgst ${ }_{d}^{p}$ to $c g s t$ (line 441 ). Now the replica stops accepting updates with timestamps $<$ curr_cgst $_{d}^{p}$ (line 26 . Then it sends a signed COLLECT_ACK (cgst, st) message back to $r_{i}^{p}$ (line 443, where $s t$ is the set of updates with timestamps $\leq$ curr_cgst $_{d}^{p}$ in its store ${ }_{d}^{p}$ (line 442 ).

The replica $r_{d}^{p}$ waits to receive a set, denoted store, of COLLECT_ACK messages from a quorum $Q^{\prime}$ of $\operatorname{REPLICAS}(p)$. We require the messages in store carry the same curr_cgst as in the corresponding COLLECT message and the signatures of all the collected updates be valid (i.e., safe_collect(store) holds). Then, it sends a signed PROPOSE message with store as its proposal to REPLICAS $(p)$ (line 421).

When replica $r_{d}^{p}$ receives a PROPOSE message from replica $r_{i}^{p}$, it also checks the predicate safe_collect(store) (line 423). After setting curr_cgst ${ }_{d}^{p}$ to store, it sends a signed PREPARED message to $\operatorname{REPLICAS}(p)$. When replica $r_{d}^{p}$ receives a set $C$ of PREPARED messages from a quorum $Q$ of $\operatorname{REPLICAS}(p)$, both its curr_view $_{d}^{p}$ and curr_store ${ }_{d}^{p}$ are prepared (lines $4 \mid 29$ and $4 \mid 30$ ). The certification $C$ is also remembered in cert ${ }_{d}^{p}$ (line 431 . They will be sent to new leaders in view changes to ensure agreement across views (line 410). Then the replica sends a signed COMMit message to $\operatorname{REPLICAS}(p)$ (line 432 ).

When replica $r_{d}^{p}$ receives a set of COMMIT message from a quorum $Q$ of $\operatorname{REPLicAs}(p)$, it computes store as the union of the sets of updates $s t_{i}$ collected from each $r_{i}^{p}$ in curr_store ${ }_{d}^{p}$ (line 4 35. If cgst $_{d}^{p}$ is smaller than the cgst in curr_store ${ }_{d}^{p}$, the replica sets cgst ${ }_{d}^{p}$ to this cgst (line 436 , and replaces the set of updates with timestamps $\leq$ curr_cgst $_{d}^{p}$ in store ${ }_{d}^{p}$ with the new store (line 438).

\section{Evaluation}

We evaluate Byz-GentleRain in terms of performance, throughput, and latency of remote update visibility. We also compare Byz-GentleRain to Byz-RCM.

\subsection{Implementation and Setup}

We implement both Byz-GentleRain and Byz-RCM in Java and use Google's Protocol Buffers ${ }^{6}$ for message serialization. We implement the key-value stores as HashMap, where each key is associated with a linked list of versioned values. The key-value stores hold 300 keys in main memory, with each key of size 8 bytes and each value of size 64 bytes.

We run all experiments on 4 Aliyun 7 instances running Ubuntu 16.04. Each instance is configured as a data center, with 1 virtual CPU core, $300 \mathrm{MB}$ memory, and 1G SSD storage. All keys are shared into 3 partitions within each data center, according to their hash values.

\footnotetext{
${ }^{6}$ Protocol Buffers: https://developers.google.com/protocol-buffers

7 Alibaba Cloud: https://www.alibabacloud.com/
} 


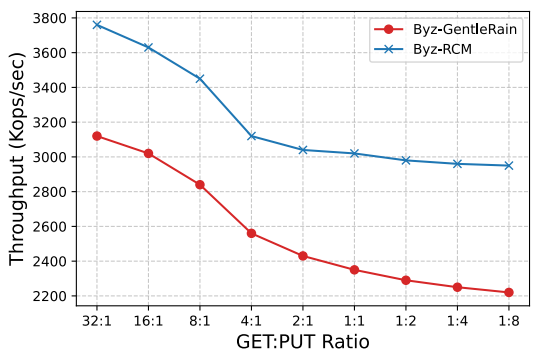

(a) Throughput

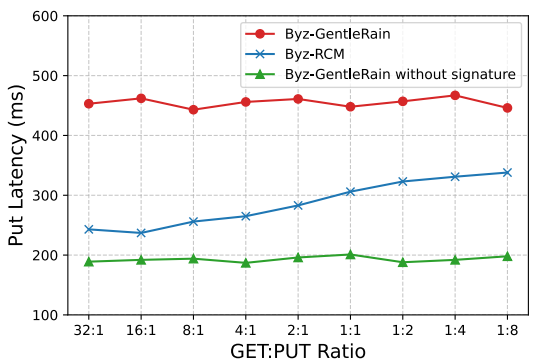

(c) PUT latency

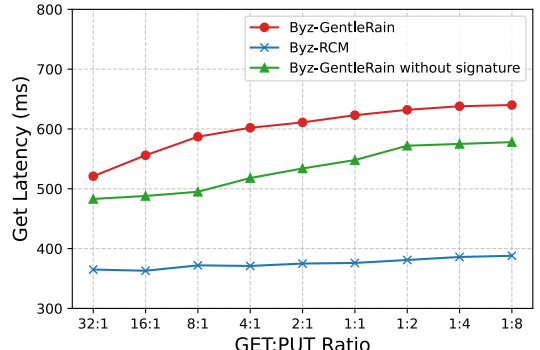

(b) GET latency

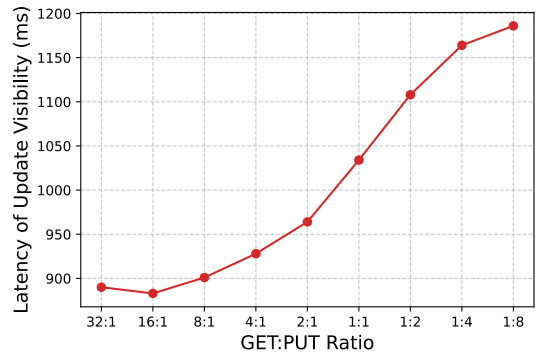

(d) PUT visibility

Fig. 2: Evaluation of Byz-GentleRain and Byz-RCM in failure-free scenarios.

\subsection{Evaluation Results}

Figure 2 shows the system throughput and the latency of GET and PUT operations of both Byz-GentleRain and Byz-RCM in failure-free scenarios. We vary the GET : PUT ratios of workloads. First, Byz-RCM performs better than Byz-GentleRain, especially with low GET : PUT ratios. This is because ByzRCM assumes Byzantine fault-free clients and is signature-free. In contrast, Byz-GentleRain requires clients sign each PUT_REQ request. Second, it demonstrates that Byz-GentleRain is quite efficient on typical workloads, especially for read-heavy workloads. Third, the performance of Byz-GentleRain is closely comparable to that of Byz-RCM, if digital signatures are omitted deliberately from Byz-GentleRain; see Figures 2b and 2c. Finally, Figure 2d shows the latency of PUT visibility, which gets higher and higher with more and more PUT operations.

We also evaluate Byz-GentleRain in several typical Byzantine scenarios. Generally, both Byzantine clients and replicas may fail by crash or send arbitrary messages. Particularly, we consider (1) Byzantine clients that may send GET_REQ and/or PUT_REQ requests with incorrect timestamps (line 14 and line 112, and (2) Byzantine replicas that may broadcast different global stable time cgst to replicas in different partitions (line 311 ).

Figure 3 demonstrates the impacts of various Byzantine failures on the system throughput of Byz-GentleRain. On the one hand, the Byzantine failures of types 


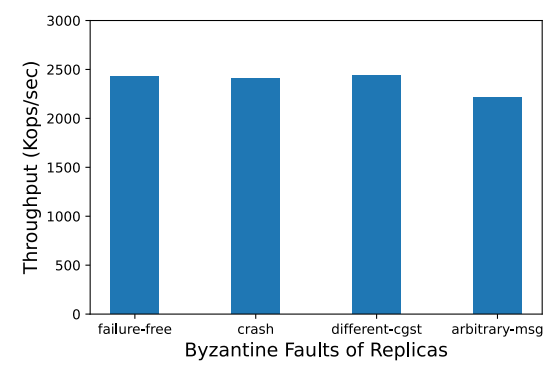

(a)

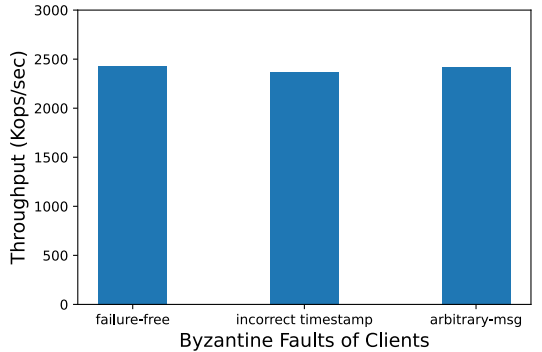

(b)

Fig. 3: Evaluation of Byz-GentleRain in Byzantine scenarios.

(1) and (2) above has little impact on throughput. On the other hand, frequently sending arbitrary messages, such as NEW_CGST or PROPOSE messages, does hurt throughput. This is probably due to the signatures carried by these messages.

\section{Related Work}

As far as we know, Byz-RCM [19 is the only causal consistency protocol that considers Byzantine faults. It achieves causal consistency in the client-server model with $3 f+1$ servers where up to $f$ servers may suffer Byzantine faults, and any number of clients may crash. Byz-RCM has also been shown optimal in terms of failure resilience. However, Byz-RCM did not tolerate Byzantine clients, and thus it could rely on clients' requests to identify bogus requests from Byzantine servers 19 .

Linde et. al. [14] consider peer-to-peer architecture. A centralized server maintains the application data, while clients replicate a subset of data and can directly communicate with each other. They analyze the possible attacks of clients to causal consistency (the centralized server is assumed correct), derive a secure form of causal consistency, and propose practical protocols for implementing it.

Liskov and Rodrigues extend the notion of linearizability [10 and define BFT-linearizability in the presence of Byzantine servers and clients 15. They also design protocols that achieve BFT-linearizability despite Byzantine clients. The protocols require $3 f+1$ replicas of which up to $f$ replicas may be Byzantine. They are quite efficient for linearizable systems: Writes complete in two or three phases, while reads complete in one or two phases.

Auvolat et. al. [3] defines a Byzantine-tolerant Causal Order broadcast (BCObroadcast) abstraction and proposes an implementation for it. However, as a communication primitive for replicas, BCO-broadcast does not capture the get/put semantics from the perspective of clients. Thus, it does not prevent Byzantine clients from violating causality. 


\section{Conclusion}

We present Byz-GentleRain, the first causal consistency protocol which tolerates up to $f$ Byzantine servers among $3 f+1$ servers in each partition and any number of Byzantine clients. The preliminary experiments show that Byz-GentleRain is quite efficient on typical workloads. Yet, more extensive large-scale experiments on more benchmarks are needed. We will also explore optimizations of our synchronization protocol in Algorithm 4 in future work.

\section{Acknowledgements}

This work was partially supported by the CCF-Tencent Open Fund (CCFTencent RAGR20200124) and the National Natural Science Foundation of China (No. 61772258).

\section{References}

1. Ahamad, M., Neiger, G., Burns, J.E., Kohli, P., Hutto, P.W.: Causal memory: Definitions, implementation, and programming. Distributed Computing 9(1), 3749 (1995)

2. Akkoorath, D.D., Tomsic, A.Z., Bravo, M., Li, Z., Crain, T., Bieniusa, A., Preguiça, N., Shapiro, M.: Cure: Strong semantics meets high availability and low latency. In: IEEE 36th International Conference on Distributed Computing Systems (ICDCS). pp. 405-414 (2016)

3. Auvolat, A., Frey, D., Raynal, M., Taïani, F.: Byzantine-tolerant causal broadcast. Theoretical Computer Science (2021)

4. Bouajjani, A., Enea, C., Guerraoui, R., Hamza, J.: On verifying causal consistency. In: Proceedings of the 44th ACM Symposium on Principles of Programming Languages. p. 626-638. POPL 2017 (2017)

5. Bravo, M., Chockler, G.V., Gotsman, A.: Making byzantine consensus live. In: 34th International Symposium on Distributed Computing, DISC 2020. pp. 23:1-23:17 (2020). https://doi.org/10.4230/LIPIcs.DISC.2020.23

6. Brewer, E.A.: Towards robust distributed systems (abstract). In: Proceedings of the 19th Annual ACM Symposium on Principles of Distributed Computing. pp. 7-. PODC'00 (2000)

7. Burckhardt, S., Gotsman, A., Yang, H., Zawirski, M.: Replicated data types: Specification, verification, optimality. In: Proceedings of the 41st ACM Symposium on Principles of Programming Languages. pp. 271-284. POPL '14 (2014)

8. de Castro, M.O.T.: Practical Byzantine fault tolerance. Ph.D. thesis, Massachusetts Institute of Technology, Cambridge, MA, USA (2000)

9. Du, J., Iorgulescu, C., Roy, A., Zwaenepoel, W.: Gentlerain: Cheap and scalable causal consistency with physical clocks. In: Proceedings of the ACM Symposium on Cloud Computing. p. 1-13. SoCC '14 (2014)

10. Herlihy, M.P., Wing, J.M.: Linearizability: A correctness condition for concurrent objects. ACM Trans. Program. Lang. Syst. 12(3), 463-492 (Jul 1990). https://doi.org/10.1145/78969.78972 
11. Jiang, X., Wei, H., Huang, Y.: A generic specification framework for weakly consistent replicated data types. In: International Symposium on Reliable Distributed Systems (SRDS). pp. 143-154 (2020)

12. Lamport, L.: Time, clocks, and the ordering of events in a distributed system. Commun. ACM 21(7), 558-565 (Jul 1978)

13. Lesani, M., Bell, C.J., Chlipala, A.: Chapar: Certified causally consistent distributed key-value stores. In: Proceedings of the 43rd Annual ACM Symposium on Principles of Programming Languages. p. 357-370. POPL '16 (2016)

14. van der Linde, A., Leitão, J.a., Preguiça, N.: Practical client-side replication: Weak consistency semantics for insecure settings. Proc. VLDB Endow. 13(12), 2590-2605 (Jul 2020). https://doi.org/10.14778/3407790.3407847

15. Liskov, B., Rodrigues, R.: Tolerating byzantine faulty clients in a quorum system. In: 26th IEEE International Conference on Distributed Computing Systems (ICDCS'06). pp. 34-34 (July 2006). https://doi.org/10.1109/ICDCS.2006.83

16. Lloyd, W., Freedman, M.J., Kaminsky, M., Andersen, D.G.: Don’t settle for eventual: Scalable causal consistency for wide-area storage with cops. In: Proceedings of the 23rd ACM Symposium on Operating Systems Principles. pp. 401-416. SOSP '11 (2011)

17. Lloyd, W., Freedman, M.J., Kaminsky, M., Andersen, D.G.: Stronger semantics for low-latency geo-replicated storage. In: Proceedings of the 10th USENIX Conference on Networked Systems Design and Implementation. pp. 313-328. NSDI'13 (2013)

18. Perrin, M., Mostefaoui, A., Jard, C.: Causal consistency: Beyond memory. In: Proceedings of the 21st ACM Symposium on Principles and Practice of Parallel Programming. PPoPP '16 (2016)

19. Tseng, L., Wang, Z., Zhao, Y., Pan, H.: Distributed causal memory in the presence of byzantine servers. In: 18th IEEE International Symposium on Network Computing and Applications, NCA 2019. pp. 1-8 (2019)

20. Tyulenev, M., Schwerin, A., Kamsky, A., Tan, R., Cabral, A., Mulrow, J.: Implementation of cluster-wide logical clock and causal consistency in mongodb. In: Proceedings of the 2019 International Conference on Management of Data. pp. 636-650. SIGMOD '19 (2019) 
Table A1: Predicates in Algorithm 4 (adapted from 5]).

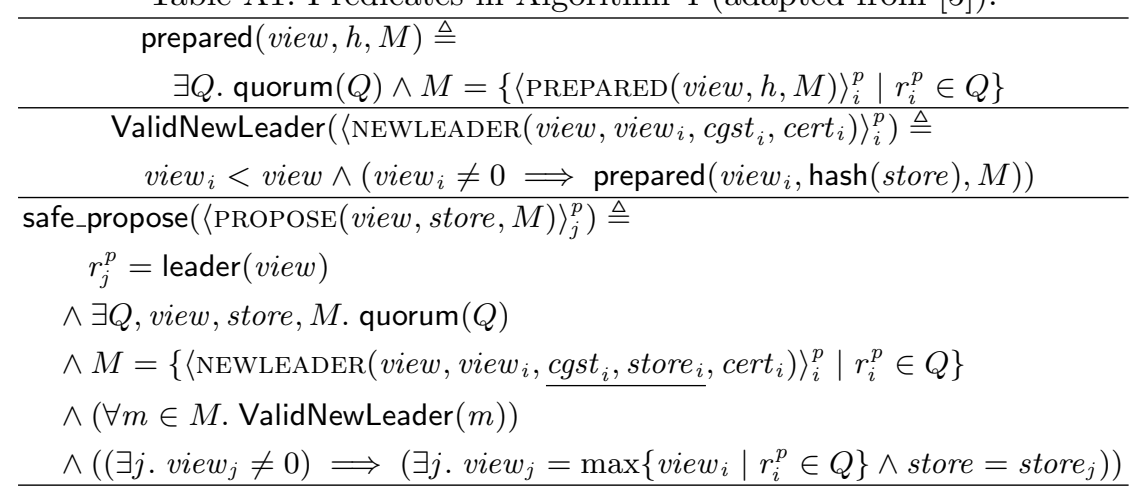

\section{A Correctness of Byz-GentleRain}

We show that Byz-GentleRain satisfies Byz-CC. We assume that single-shot PBFT is correct and refer its detailed correctness proof to 51. Table A1 gives the definitions of the predicates ValidNewLeader and safe_propose used in Algorithm 4, which are also adapted from [5].

Remark 1. In the following, we use $R$ and $W$ to denote the set of GET and PUT operations, respectively. We also define $O \triangleq R \cup W$ to denote the set of all operations.

For a variable, e.g., clock $_{c}$ at client $c$, we refer to its value at time $\sigma$ by, e.g., $\left(\text { clock }_{c}\right)_{\sigma}$.

According to the description of Algorithms 1 and 2 ,

Lemma 1. Rule I-Rule III are maintained by Byz-GentleRain.

Lemma 2. Inv I-Inv III are maintained by Byz-GentleRain.

Proof. Inv 1 holds due to line 1 11. Inv II holds due to the read rule at line 26 . By the correctness of single-shot PBFT [5], Inv III holds.

Definition 1 (Timestamps). We use ts(o) to denote the timestamp of operation o, which is defined as follows:

- For a GET operation o, ts(o) refers to the value of "ts" at line 1 12.

- For a PUT operation o, ts(o) refers to the value of "cl" at line 11:12.

\section{Lemma 3.}

$$
\left(w \stackrel{\text { so }}{\longrightarrow} w^{\prime} \wedge w \in W \wedge w^{\prime} \in W\right) \Longrightarrow \operatorname{ts}\left(w^{\prime}\right)>t s(w) .
$$

Proof. Suppose that $w^{\prime}$ is issued by client $c$ at time $\sigma^{\prime}$.

$$
\operatorname{ts}(w) \leq\left(\mathrm{dt}_{c}\right)_{\sigma^{\prime}}<\left(\operatorname{clock}_{c}\right)_{\sigma^{\prime}}=\operatorname{ts}\left(w^{\prime}\right) .
$$




\section{Lemma 4.}

$$
(r \stackrel{\text { so }}{\longrightarrow} w \wedge r \in R \wedge w \in W) \Longrightarrow \operatorname{ts}(w)>\operatorname{ts}(r) .
$$

Proof. Suppose that $r$ and $w$ are issued by correct client $c$ at time $\sigma_{r}$ and $\sigma_{w}$, respectively. By line 12 .

$$
\operatorname{ts}(r)=\max \left\{\left(\operatorname{cgst}_{c}\right)_{\sigma_{r}},\left(\mathrm{dt}_{c}\right)_{\sigma_{r}}\right\} .
$$

By Inv [

$$
\operatorname{ts}(w)>\left(\operatorname{cgst}_{c}\right)_{\sigma_{w}}>\left(\operatorname{cgst}_{c}\right)_{\sigma_{r}} .
$$

Moreover,

$$
\operatorname{ts}(w)>\left(\mathrm{dt}_{c}\right)_{\sigma_{w}} \geq\left(\mathrm{dt}_{c}\right)_{\sigma_{r}} .
$$

Putting it together yields

$$
\operatorname{ts}(w)>t s(r)
$$

\section{Lemma 5.}

$$
(w \stackrel{\text { so }}{\longrightarrow} r \wedge w \in W \wedge r \in R) \Longrightarrow \operatorname{ts}(r) \geq \operatorname{ts}(w) .
$$

Proof. Suppose that $r$ are issued by correct client $c$ at time $\sigma$. By line 12 ,

$$
\operatorname{ts}(r)=\max \left\{\left(\operatorname{cgst}_{c}\right)_{\sigma},\left(\mathrm{dt}_{c}\right)_{\sigma}\right\} \geq\left(\mathrm{dt}_{c}\right)_{\sigma} .
$$

By line 115.

$$
\left(\mathrm{dt}_{c}\right)_{\sigma} \geq t s(w)
$$

Thus,

$$
\operatorname{ts}(r) \geq \operatorname{ts}(w)
$$

\section{Lemma 6.}

$$
\left(r \stackrel{\text { so }}{\longrightarrow} r^{\prime} \wedge r \in R \wedge r^{\prime} \in R\right) \Longrightarrow t s\left(r^{\prime}\right) \geq t s(r) .
$$

Proof. Suppose that $r$ and $r^{\prime}$ are issued by correct client $c$ at time $\sigma$ and $\sigma^{\prime}$, respectively. By line 12 .

$$
\operatorname{ts}(r)=\max \left\{\left(\operatorname{cgst}_{c}\right)_{\sigma},\left(\mathrm{dt}_{c}\right)_{\sigma}\right\},
$$

and

$$
\operatorname{ts}\left(r^{\prime}\right)=\max \left\{\left(\operatorname{cgst}_{c}\right)_{\sigma^{\prime}},\left(\mathrm{dt}_{c}\right)_{\sigma^{\prime}}\right\}
$$

Moreover,

$$
\left(\operatorname{cgst}_{c}\right)_{\sigma^{\prime}} \geq\left(\operatorname{cgst}_{c}\right)_{\sigma} \wedge\left(\mathrm{dt}_{c}\right)_{\sigma^{\prime}} \geq\left(\mathrm{dt}_{c}\right)_{\sigma} .
$$

Thus,

$$
\operatorname{ts}\left(r^{\prime}\right) \geq \operatorname{ts}(r)
$$

\section{Lemma 7.}

$$
o \stackrel{\text { so }}{\longrightarrow} o^{\prime} \wedge o \in O \wedge o^{\prime} \in O \Longrightarrow \operatorname{ts}\left(o^{\prime}\right) \geq \operatorname{ts}(o) .
$$


Proof. By Lemmas 36 .

\section{Lemma 8.}

$$
w \stackrel{\text { rf }}{\rightarrow} r \wedge w \in W \wedge r \in R \Longrightarrow \operatorname{ss}(r) \geq t s(w) .
$$

Proof. By the read rule at line 27.

\section{Lemma 9.}

$$
o \rightsquigarrow o^{\prime} \wedge o \in O \wedge o^{\prime} \in O \Longrightarrow \operatorname{ts}\left(o^{\prime}\right) \geq \operatorname{ts}(o) .
$$

Proof. By Lemmas 7 and 8 .

Lemma 10. Consider $r \in R$ and $w \in W$. Suppose $r$ reads from some value at a correct replica $r_{d}^{p}$ at time $\sigma$ (line 23). If $w$ would be added to store $_{d}^{p}$ at a later time than $\sigma$ (line 29) then $\operatorname{ts}(w)>\operatorname{ts}(r)$.

Proof. By Rule I.

$$
\left(\operatorname{cgst}_{d}^{p}\right)_{\sigma} \geq \operatorname{ts}(r)
$$

By INV III.

$$
\operatorname{ts}(w)>\left(\operatorname{cgst}_{d}^{p}\right)_{\sigma} .
$$

Thus,

$$
\operatorname{ts}(w)>t s(r) .
$$

Lemma 11. Consider $r \in R$ and $w \in W$. Suppose the successful $r$ returns at time $\sigma$ (line 203) and the successful $w$ starts at a later time than $\sigma$ in partition p. Then $\neg(w \rightsquigarrow r)$.

Proof. By lines 1 and 113 , there is a correct replica at which $r$ obtains its value (line 233 before $w$ is added to the store (line 299). By Lemma 10.

$$
t s(w)>t s(r) .
$$

By Lemma 9

$$
\neg(w \rightsquigarrow r) .
$$

Theorem 1. Byz-GentleRain satisfies Byz-CC. That is, when a certain PUT operation is visible to a client, then so are all of its causal dependencies.

Proof. By Lemmas 11 and 15 .

Lemma 12. Suppose a PUT operation $w$ successfully returns in partition $p$ at time $\sigma$ (line 1 13). Then, it will eventually be in store $_{i}^{p}$ for each correct data center $i$.

Proof. By line 113 and line 420 there is a correct replica in partition $p$ at which $w$ is added to the store (line 29 before it is sent to the PBFT leader in the COLLECT_ACK message (line 443. By the correctness of single-shot PBFT [5], it will eventually be in store ${ }_{i}^{p}$ for each correct data center $i$. 
Lemma 13. Let $w$ be a successful PUT operation. Then, eventually for each correct replica $r_{i}^{j}(1 \leq i \leq D, 1 \leq j \leq P), \operatorname{cgst}_{i}^{j} \geq t s(w)$.

Proof. Suppose $w$ successfully returns in partition $p$. Then it is added to the stores of at least $f+1$ correct replicas in $\operatorname{Replicas}(p)$. By Algorithm 3 , eventually for each correct replica $r_{i}^{j}$, $\operatorname{Ist}_{i}^{j} \geq t s(w)$ and $\operatorname{gst}_{i}^{j} \geq t s(w)$. By Algorithm 4 . $\operatorname{cgst}_{i}^{j} \geq t s(w)$.

Lemma 14. Suppose a correct replica sends a NEW_CGST(gst) message (line 311). Then, there is a cgst $\geq$ gst such that eventually for each correct replica $r_{i}^{J}$ $(1 \leq i \leq D, 1 \leq j \leq P), \operatorname{cgst}_{i}^{j} \geq$ cgst.

Proof. A byzantine replica can propose $g s t_{d}^{p}$ to replica $r_{d}^{p}$, but it will take effect only when $g s t_{d}^{p}<l s t_{d}^{p}$ maintained by $r_{d}^{p}$. If $g s t_{d}^{p}$ is smaller than a $g s t^{\prime}$ broadcast by a correct replica, then it will be overwritten at $r_{d}^{p}$. And eventually, there must be a $g s t$ version $\geq r_{d}^{p}$ broadcast by a correct replica. Thus it will eventually by overwritten by a correct $g s t$ version.

Lemma 15. Consider $r \in R$ and $w \in W$. Suppose the successful $r$ returns at time $\sigma$ (line 203) and the successful $w$ starts at a later time than $\sigma$ in any partition $j \neq p$. Then $\neg(w \rightsquigarrow r)$.

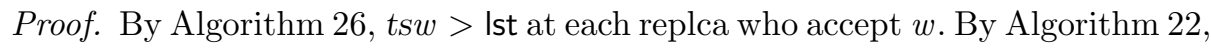
$t s r \leq$ cgst at each replica who reply it. Since lst at any correct correct server is an upbound of all the cgst at all correct replicas, $\operatorname{tsof}(w)>$ lst $>$ cgst $>\operatorname{ss}(r)$. So $\neg(w \rightsquigarrow r)$. 